\title{
Variety in magnetic resonance imaging of enlarged perivascular space: Case reports
}

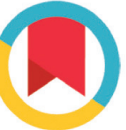

CrossMark
Indonesian Journal of Neurosurgery

'Department of Neurosurgery, Graduate School of Medical and Dental Sciences, Kagoshima University, Kagoshima,

Japan;

${ }^{2}$ Department of Neurosurgery, Medical Faculty, Universitas Diponegoro,

Semarang, Indonesi;

${ }^{3}$ Department of Neurosurgery, AWS Hospital, and Medical School, Universitas Mulawarman, Samarinda, Indonesia;

*Corresponding author: Mohammad Ali Akbar;

Department of Neurosurgery, Graduate School of Medical and Dental Sciences, Kagoshima University, Kagoshima,

Japan;

aliiakbarr30@gmail.com

Received: 2021-02-27

Accepted: 2021-10-20

Published: 2021-12-03

\author{
Mohammad Ali Akbar ${ }^{1,2^{*}}$, Muhammad Kamil' ${ }^{1}$ F. M. Moinuddin' ${ }^{1}$, \\ Shingo Fujio', Hirofumi Hirano ${ }^{1}$, Arie Ibrahim ${ }^{3}$, Yuriz Bakhtiar², \\ Thohar Arifin², Zainal Muttaqin², Kazunori Arita ${ }^{1}$
}

\section{ABSTRACT}

Introduction: Expansion of perivascular spaces leads to tissue damage in the surrounding parenchyma. Enlarged perivascular spaces (EPS) have been long regarded as benign and normal variants, and have been subject to little investigation. We report three cases of EPS in unusual locations with their typical findings and clinical symptoms as consideration to differentiate them from other brain lesions.

Case presentation: The first case was a 64-year-old woman presenting with vertigo, vomiting, and shoulder stiffness. The second case was a 25-year-old woman who worked as an agricultural trainee who complained of intermittent headaches 3 years before admission. The third case was a 42-year-old woman with dizziness and recurring headache. The MRI finding in T1 flair was a hypointense, which was surrounded by a hyperintensity wall. T2WI revealed a hyperintensity in the superior temporal lobe, with no pathological finding to prove an ischemic or infarction process in Diffuse Weighted Imaged (DWI) and Apparent Diffusion Coefficient (ADC) images.

Conclusion: These 3 locations in our case reports should be one of consideration for diagnosing an EPS with its typical feature. A routine MRI study is needed to follow up progressivity of EPS.

Keywords: enlarged perivascular space, magnetic resonance imaging, Virchow-Robin space.

Cite This Article: Akbar, M.A., Kamil, M., Moinuddin, F.M., Fujio, S., Hirano, H., lbrahim, A., Bakhtiar, Y., Arifin, T., Muttaqin, Z., Arita, K. 2021. Variety in magnetic resonance imaging of enlarged perivascular space: Case reports. Indonesian Journal of Neurosurgery 4(3): 87-90. D0I: 10.15562/ijn.v4i3.85

\section{INTRODUCTION}

Perivascular space, also known as Virchow-Robin space, is an invagination of the subarachnoid space that surrounds the vessel wall as it courses from the subarachnoid space through the brain parenchyma. ${ }^{1}$ The spaces surround the perforating arteries, arterioles, veins, and venules, but not the capillaries of the central nervous system. The spaces are normally microscopic, but when dilated, they may be seen on magnetic resonance imaging (MRI). The first finding of dilatation of these perivascular spaces was described by Durand-Fardel. They may occur in the basal ganglia or the white matter. $^{2}$

Because the expansion of perivascular spaces leads to tissue damage in the surrounding parenchyma, enlarged perivascular spaces (EPS) have been long regarded as benign and normal variants, and have been subject to little investigation. $^{3}$ They mimic a cystic lesion of the brain parenchyma and pose an important diagnostic challenge due to its neuroimaging findings. ${ }^{4}$ EPS is related to extensive white matter lesions also known as leukoaraiosis in computerized tomography (CT) imaging, hypertension, and depression in the elderly, which pointing an association between perivascular spaces and cerebral microvessel disease. ${ }^{5}$

We reported three cases of EPS in unusual locations with their typical findings and clinical symptoms as consideration to differentiate them from other brain lesions.

\section{CASE PRESENTATION}

\section{Case 1}

A 64-year-old woman presenting with vertigo, vomiting, and shoulder stiffness underwent an MRI study on 19 March 2014, which revealed a cyst-like lesion on the tip of the left temporal lobe with perifocal hyperintensity on a T2 weighted image (T2WI). The clinical neurologic and neuropsychological examination findings were normal, so the patient was followed up by a routine MRI. In October 2014, the MRI showed an increase in the intensity of the lesion, so she was referred to our department (Fig. 1). This patient had no complaint, but she had a multi cerebral infarction around ten years ago. She was under aspirin therapy for cerebrovascular disease.

The MRI finding in T1 flair was a hypointense, which was surrounded by a hyperintensity wall (Fig. 2B). T2WI found a hyperintensity in the superior temporal lobe (Fig. 2A), with no pathological finding to prove an ischemic or infarction process in Diffuse Weighted Imaged (DWI) and Apparent Diffusion Coefficient (ADC) images (Fig. 3). MR spectroscopy study showed an increasing $\mathrm{N}$-acetyl aspartate and choline level but the Cho/NAA ratio 

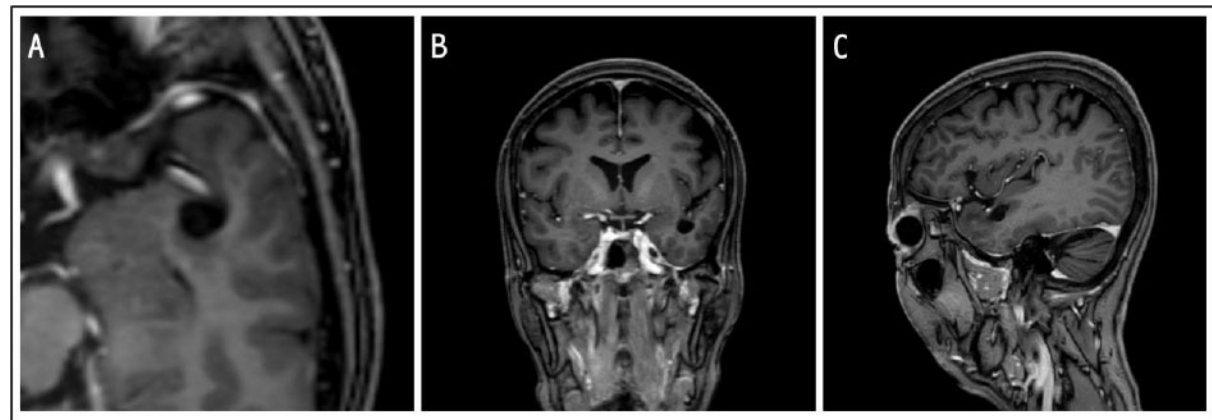

Figure 1. T1W brain MRI. A. Axial, B. Coronal, and C. Sagittal sections showed a round shape hypointense lesion on the left temporal lobe, with a sharp margin and with a signal void entering the lesion.
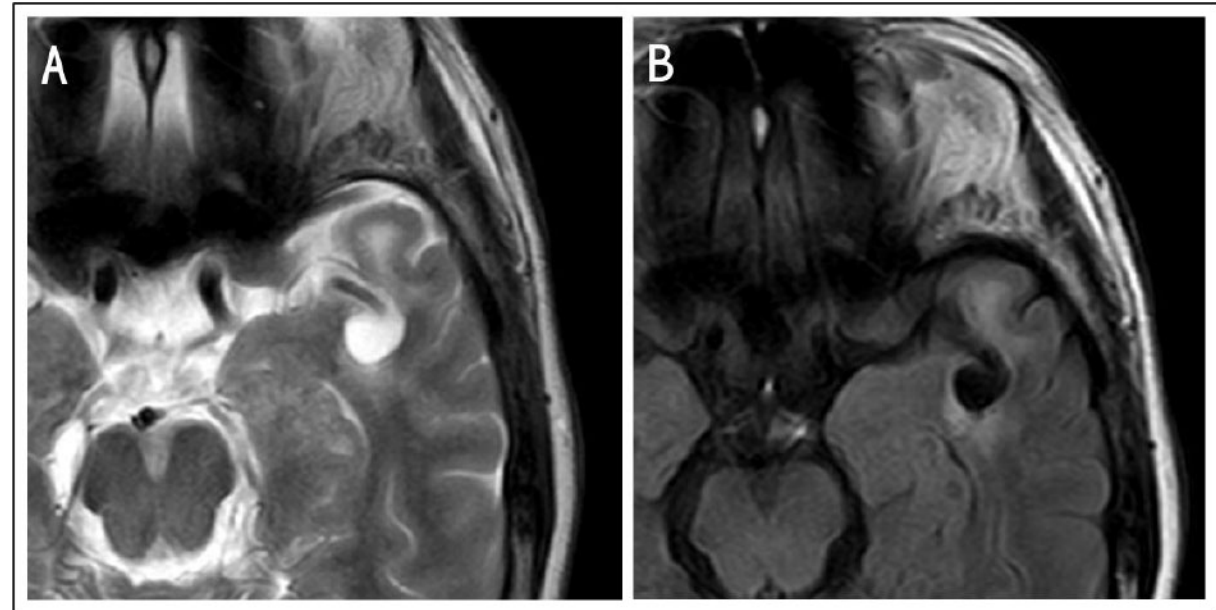

Figure 2. A. On axial T2W brain MRI, the lesion was hyperintense. B. On axial FLAIR brain MRI, the intensity of the lesion was similar with cerebrospinal fluid and there was hyperintensity that surrounded the mass.
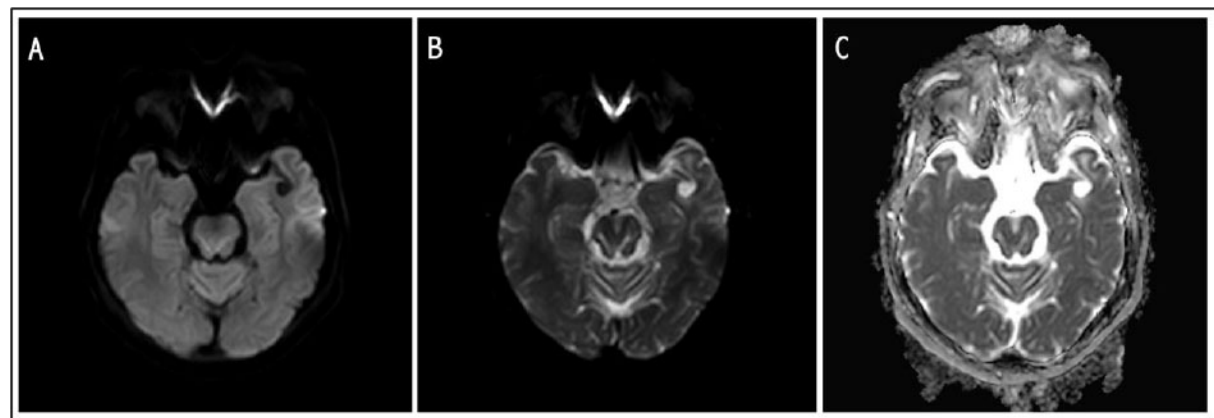

Figure 3. DWI, ADC showed no evidence of ischaemic.

was still normal, suggesting benign lesion than malignancy. Under this MRI finding, this patient was diagnosed as dilated perivascular space in the left superior temporal lobe.

\section{Case 2}

A 25-year-old woman who worked as an agricultural trainee complained of intermittent headaches 3 years before admission. She underwent an MRI study in January 2015, which revealed multiple small cyst-like lesions in the left frontal lobe with no mass effect. The laboratory result showed no evidence of infection, and the clinical neurologic and neuropsychological examination findings were normal. The presence of multiple cysts on the left frontal lobe was suggesting dilated perivascular space rather than a brain tumor or parasitic infection.

The MRI finding in T1 flair was a hypointense, which was surrounded by a hyperintensity wall. T2WI revealed multiple hyperintensities in the left frontal lobe (Fig. 4), with no pathological finding to prove an ischemic or infarction process in DWI and ADC images. MR spectroscopy study showed no abnormality of the brain metabolites. Under this MRI finding, this patient was diagnosed as dilatated perivascular space in the left frontal lobe and was followed up with a routine MRI study.

\section{Case 3}

A 42-year-old woman was referred to us for further study due to her dizziness and recurring headache. She had hypertension that was controlled with antihypertensive. She did not have any history of exposure to pets or farm animals. Physical examinations, including neurological examination, were within normal limits. No evidence of papilledema was found on fundus examination.

The MRI T2WI disclosed multiple round ovoid exposures in the cerebral peduncle (coronal section) and around the substansia nigra on the axial section. The signal intensities were identical to those of cerebrospinal fluid (CSF) with all pulse sequences (Fig. 5). No abnormality was found in DWI and ADC and the MR spectroscopy revealed normal.

\section{DISCUSSION}

The appearance of normal perivascular space in the MRI of the brain is typical, so can be differentiated with cystic neoplasm, lacunar infarction, and other brain lesions by using radiological findings. They are round or oval, have smooth margins, are generally less than $2 \mathrm{~mm}$ (some authors considered less than $5 \mathrm{~mm}$ as normal) in diameter, and are located along the path of penetrating blood vessels. ${ }^{1}$ T2WI is considered a sensitive method for depicting perivascular spaces with an isointense with CSF on all MRI sequences. The most common location of PVSs is along lenticulostriate arteries, adjacent to the basal ganglia, above the anterior perforated substance, and adjacent to 


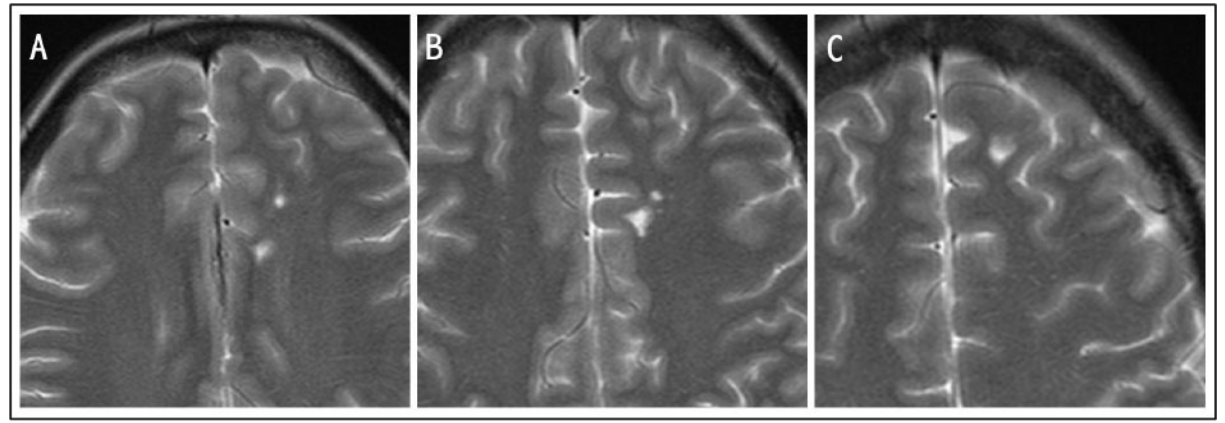

Figure 4. Axial T2W brain MRI showed a multiple round shape hyperintense lesion on the left frontal lobe.

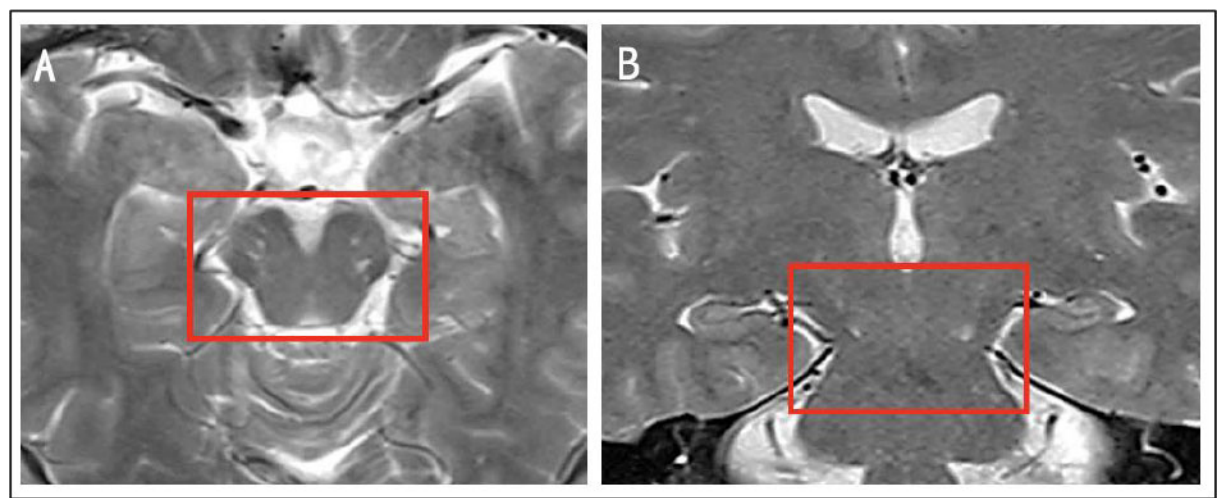

Figure 5. T2 Axial and coronal section disclosed multiple round ovoid hyperintensities in the cerebral peduncle (coronal section) and around substansia nigra on the axial section.

the anterior commissure, followed by the midbrain, subcortical white matter subinsular cortex, and extreme capsule. ${ }^{6}$ Less common areas include the thalami, cerebellum, corpus callosum, cingulate gyrus, and temporal lobe.

Eighty percent of EPS occurs in the elderly. The highest prevalence of EPS occurs in people aged $61-80$ years old and is mostly located in the high convexity site of the brain, and it has been considered as a normal part of the aging process due to atrophy or a normal variant. ${ }^{8}$ In young healthy subjects, EPS has been described in association with several diseases including metabolic and genetic disorders, vascular pathologies, neuroectodermal syndromes, chronic alcoholism, parkinsonism, autism, multiple sclerosis, and mild traumatic brain injuries. ${ }^{7}$ Other risk factors such as hypertension and small vessel disease were also associated with EPS located in basal ganglia rather than for those located in the white matter. ${ }^{9}$

In case 1, we found an EPS in the superior anterior temporal lobe with a perilesional high-intensity appearance. The feature of perilesional high intensity that was seen in this case cannot exclude the EPS diagnosis because EPS compression of the adjacent parenchymal vessels accelerated white matter ischemic change. ${ }^{10}$ Rawal et al. reported about 15 cases selections with the anterior superior temporal subcortical white matter may exhibit a regional preference for EPS.11 Salzman et al. observed giant perivascular spaces in the frontal lobe occurring in 8 cases from 37 cases, and temporal predominance of a subcortical lesion was only found in 2 cases from 37 cases with giant perivascular spaces. Subcortical white matter in the frontal lobe may exhibit a regional predilection of EPS. ${ }^{6}$

Richardson et al. identified punctate and linear foci of T2W MR images in the midbrain of $32(20.4 \%)$ of 157 patients undergoing high-resolution MR imaging of the brainstem. The most common site of EPS in the midbrain was in the pontomesenchepalicjunction surrounding the penetrating branches of the collicular and accessory artery. In our case, we disclosed a high focal signal isointense with CSF in the cerebral peduncle in the axial section. However, a study of perivascular in midbrain conducted by Saeki et al. found perivascular spaces less frequent in the cerebral peduncle on the axial section. ${ }^{12}$ This was because the anatomic structure of the collicular and accessory arteries, which are responsible for giving numerous penetrating branches in the cerebral peduncle and the substansia nigra, has a steeply oblique course through the brainstem, accounting for their segmental visualization on serial MR images. ${ }^{13}$

The most common presenting symptomsarenonspecific, such asheadache and dizziness. But in a specific location, it may cause a serious medical problem. Association between EPS in cerebral white matter with cognitive dysfunction has been proved as a manifestation of cerebral white matter lesions..$^{14}$ Giant EPS can cause a mass effect. Other symptoms, including poor concentration, memory impairment, and dementia, have also been reported to be associated with EPS in the frontal lobe..$^{15}$ In the midbrain, EPS can cause hydrocephalus due to aqueductal stenosis. ${ }^{16}$ Motor weakness and parkinsonism were also reported as a manifestation of EPS in the midbrain. ${ }^{17,18}$ In cases of EPS with progressive symptoms and signs, especially those of huge size combined with hydrocephalus, surgical treatment is recommended. These include CSF diversion, ventriculoperitoneal shunt, cystoperitoneal shunt, ventriculocisternostomy, and implantation of a cyst catheter and reservoir. ${ }^{19}$

\section{CONCLUSION}

These three locations in our case reports should be one of consideration for diagnosing an EPS with its typical feature. A routine MRI study is needed to follow up progressivity of EPS.

\section{CONFLICT OF INTEREST}

There was no conflict of interest related to the materials or methods used in this study. 


\section{FUNDING}

This article received no specific funding from any funding agency in the public, commercial, or not-for-profit sectors.

\section{AUTHOR'S CONTRIBUTION}

Authors took part in the design of the study, contributed to data collection, participated in writing the manuscript and all agree to accept equal responsibility for the accuracy of the contents of this article.

\section{REFERENCES}

1. Braffman BH, Zimmerman RA, Trojanowski JQ, Gonatas NK, Hickey WF, Schlaepfer WW. Brain MR: Pathologic correlation with gross and histopathology. 1. Lacunar infarction and Virchow-Robin Spaces. AJR Am J Roentgenol. 1988; 151(3): 551 - 8. https://doi.org/10.2214/ ajr.151.3.551.

2. Adachi M, Hosoya T, Haku T, Yamaguchi K. Dilated Virchow-Robin spaces: MRI pathological study. Neuroradiology. 1998; 40(1): 27 - 31. https://doi.org/10.1007/ s002340050533.

3. Custer BS, Koepsell TD, Mueller BA. The association between breast carcinoma and meningioma in women. Cancer. 2002; 94(6): 1626 - 35. https://doi.org/10.1002/cncr.10410.

4. Rohlfs J, Riegel T, Khalil M, Iwinska-Zelder J, Mennel H-D, Bertalanffy H, Hellwig D. Enlarged perivascular spaces mimicking multicystic brain tumors. Report of two cases and review of the literature. J Neurosurg. 2005; 102(6): 1142 - 6. https://doi.org/10.3171/ ins.2005.102.6.1142.

5. Doubal FN, MacLullich AM, Ferguson KJ, Dennis MS, Wardlaw JM. Enlarged perivascular spaces on MRI are a feature of cerebral small vessel disease. Stroke. 2010; 41(3): 450 - 4. https://doi.org/10.1161/ STROKEAHA.109.564914.

6. Salzman KL, Osborn AG, House P, Jinkins JR, Ditchfield A, Cooper JA, Weller RO. Giant tumefactive perivascular spaces. AJNR Am J Neuroradiol. 2005; 26(2): 298 - 305.

7. Cerase A, Vallone IM, Muccio CF, Petrini C, Signori G, Venturi C. Regression of dilated perivascular spaces of the brain. Surg Radiol Anat. 2010; 32(6): 555 - 61. https://doi. org/10.1007/s00276-009-0603-y.

8. Heier LA, Bauer CJ, Schwartz L, Zimmerman RD, Morgello S, Deck MD. Large VirchowRobin spaces: MR-clinical correlation. AJNR Am J Neuroradiol. 1989; 10(5): 929 - 36.

9. Zhu YC, Tzourio C, Soumare A, Mazoyer B, Dufouil C, Chabriat H. Severity of dilated Virchow-Robin spaces is associated with age, blood pressure, and MRI markers of small vessel disease: a population-based study. Stroke. 2010; 41: 2483 - 90. https://doi.org/10.1161/ STROKEAHA.110.591586.

10. Shiratori K, Mrowka M, Toussaint A, Spalke G, Bien S. Extreme, unilateral widening of Virchow-Robin spaces: case report. Neuroradiology. 2002; 44: 990 - 2.

11. Rawal S, Croul SE, Willinsky RA, Tymianski M, Krings T. Subcortical cystic lesions within the anterior superior temporal gyrus: a newly recognized characteristic location for dilated perivascular spaces. AJNR Am J Neuroradiol. 2014; 35(2): 317 - 22. https://doi.org/10.3174/ ajnr.A3669.

12. Saeki N, Sato M, Kubota M, Uchino Y, Murai $H$, Nagai $Y$, et al. MR imaging of normal perivascular space expansion at midbrain. AJNR Am J Neuroradiol. 2005: 26(3): 566 - 71.

13. Elster AD, Richardson DN. Focal high signal on MR scans of the midbrain caused by enlarged perivascular spaces: MR-pathologic correlation.
AJR Am J Roentgenol. 156(1): 157 - 60. https:// doi.org/10.2214/ajr.156.1.1898553.

14. Maclullich AM, Wardlaw JM, Ferguson KJ, Starr JM, Seckl JR, Deary IJ. Enlarged perivascular spaces are associated with cognitive function in healthy elderly men. I Neurol Neurosurg Psychiatry. 2004; 75(11): 1519 - 23. https://doi. org/10.1136/innp.2003.030858.

15. Zhu YC, Dufouil C, Soumare A, Mazoyer B, Chabriat H, Tzourio C. High degree of dilated Virchow-Robin spaces on MRI is associated with increased risk of dementia. $J$ Alzheimers Dis. 2010; 22(2): 663 - 72. https://doi. org/10.3233/JAD-2010-100378.

16. Papayannis CE, Saidon P, Rugilo CA, Hess D, Rodriguez G, Sica REP. Expanding Virchow Robin spaces in the midbrain causing hydrocephalus. AJNR Am J Neuroradiol. 2003; 24(7): 1399 - 403

17. Romi F, Tysnes OB, Krakenes J, Savoiardo M, Aarli JA, Bindoff L. Cystic dilation of VirchowRobin spaces in the midbrain. Eur Neurol. 2002; 47: $186-8$.

18. Schroeder HW, Gaab MR, Warzok RW. Endoscopic treatment of an unusual multicystic lesion of the brainstem: case report. $\mathrm{Br} J$ Neurosurg. 1996; 10(2): 193 - 6. https://doi. org/10.1080/02688699650040368.

19. House P, Salzman KL, Osborn AG, MacDonald JD, Jensen RL and Couldwell WT. Surgical considerations regarding giant dilations of the perivascular spaces. J Neurosurg. 2004; 100(5): 820 - 4. https://doi.org/10.3171/ jns.2004.100.5.0820.

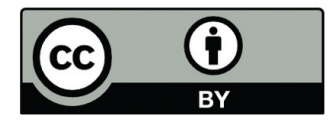

This work is licensed under a Creative Commons Attribution 